\title{
RISCOS OCUPACIONAIS DA EQUIPE DE ENFERMAGEM NO SERVIÇO DE ATENDIMENTO MÓVEL DE URGÊNCIA
}

\section{OCCUPATIONAL RISKS OF THE NURSING TEAM IN THE MOBILE EMERGENCY SERVICE}

\section{RIESGOS LABORALES DEL EQUIPO DE ENFERMERÍA DEL SERVICIO DE EMERGENCIA MÓVIL}

Jeane Santos Monteiro', Silvia Vitoria Leal Moreira Reis², Magda Rogéria Pereira Viana ${ }^{3}$

Submetido em: 15/05/2021

e25305

Aprovado em: 05/06/2021

\section{RESUMO}

Objetivo: avaliar os riscos ocupacionais apresentados pela equipe de enfermagem no serviço de atendimento móvel de urgência. Método: trata-se de uma revisão integrativa de literatura com abordagem qualitativa. A busca foi realizada nas bases de dados LILACS, Scielo, MEDLINE, Google Scholar e BVS. Para a construção da questão norteadora foi utilizada a estratégia PICo, utilizando os descritores: Riscos Ocupacionais; Serviço Médicos de Emergência; Equipe de Enfermagem. Os critérios de inclusão foram os artigos que abordassem os riscos ocupacionais; publicações com textos completos; publicados entre 2014 e 2019 e disponíveis nos idiomas Português, Inglês e Espanhol. E, os critérios de exclusão: produções incompletas, indisponíveis gratuitamente; teses; dissertações; artigos que não abordassem a temática deste estudo. Resultados: Dentre os riscos analisados, o mais frequente na literatura foi o risco ergonômico/ psicossocial, em seguida o risco biológico. Considerações finais: como limitação do estudo, observa-se a dificuldade de encontrar pesquisas com abordagem do risco mecânico com o intuito de fomentar maior conhecimento de como esse risco interfere na atividade laboral e na saúde dos profissionais da equipe de enfermagem.

PALAVRAS-CHAVE: Riscos Ocupacionais. Serviços Médicos de Enfermagem. Equipe de Enfermagem.

\begin{abstract}
Objective: to evaluate the occupational risks presented by the nursing team in the mobile emergency care service. Method: this is an integrative literature review with a qualitative approach. The search was carried out in the LILACS, SciELO, MEDLINE, Google Scholar and VHL databases. For the construction of the guiding question, the PICo strategy was used, using the descriptors: Occupational Risks; Emergency Medical Service; Nursing team. The inclusion criteria were articles that addressed occupational risks; publications with full texts; published between 2014 and 2019 and available in Portuguese, English and Spanish. And, the exclusion criteria: incomplete productions, unavailable for free; theses; dissertations; articles that did not address the theme of this study. Results: Among the risks analyzed, the most frequent in the literature was the ergonomic / psychosocial risk, followed by biological risk. Final considerations: as a limitation of the study, there is a difficulty in finding research with a mechanical risk approach in order to foster greater knowledge of how this risk interferes with the work activity and the health of the nursing team professionals.
\end{abstract}

KEYWORDS: Occupational Risks. Medical Nursing Services. Nursing team.

\footnotetext{
${ }^{1}$ Centro Universitário Santo Agostinho, Brasil. - UNIFSA

${ }^{2}$ Centro Universitário Santo Agostinho, Brasil. - UNIFSA

${ }^{3}$ Centro Universitário Santo Agostinho, Brasil. - UNIFSA
} 


\section{RECIMA21 - REVISTA CIENTÍFICA MULTIDISCIPLINAR ISSN 2675-6218}

RISCOS OCUPACIONAIS DA EQUIPE DE ENFERMAGEM NO SERVIÇO DE ATENDIMENTO MÓVEL DE URGÊNCIA Jeane Santos Monteiro, Silvia Vitoria Leal Moreira Reis, Magda Rogéria Pereira Viana

\section{RESUMEN}

Objetivo: evaluar los riesgos laborales que presenta el equipo de enfermería en el servicio móvil de urgencias. Método: se trata de una revisión integradora de la literatura con un enfoque cualitativo. La búsqueda se realizó en las bases de datos LILACS, SciELO, MEDLINE, Google Scholar y VHL. Para la construcción de la pregunta orientadora se utilizó la estrategia PICo, utilizando los descriptores: Riesgos Laborales; Servicio de Emergencias Médicas; Equipo de enfermería. Los criterios de inclusión fueron artículos que abordaran los riesgos laborales; publicaciones con textos completos; publicado entre 2014 y 2019 y disponible en portugués, inglés y español. Y, los criterios de exclusión: producciones incompletas, no disponibles de forma gratuita; tesis; disertaciones; artículos que no abordaron el tema de este estudio. Resultados: Entre los riesgos analizados, el más frecuente en la literatura fue el ergonómico / psicosocial, seguido del riesgo biológico. Consideraciones finales: como limitación del estudio, existe la dificultad de encontrar investigaciones con enfoque de riesgo mecánico para fomentar un mayor conocimiento de cómo este riesgo interfiere en la actividad laboral y en la salud de los profesionales del equipo de enfermería.

PALABRAS CLAVE: Riesgos laborales. Servicios de Enfermería Médica. Equipo de enfermería.

\section{INTRODUÇÃO}

O serviço de atendimento pré-hospitalar (APH) tem por finalidade prestar atendimento em situações de urgência e emergência clínica ou traumática, durante o transporte até uma instituição de destino, oferecendo o suporte específico para o tipo de urgência ou emergência. ${ }^{1}$

O profissional do APH atua em ambientes diversos, lidando com situações inusitadas que podem colocar sua vida em risco. Tal risco que a equipe de APH está exposta é devido aos inúmeros obstáculos que aparecem durante a sua atuação profissional, tais como: falha na qualificação técnica ou científica, treinamento inadequado, situações de difícil acesso, falta de segurança na cena, espaço diminuído para realizar procedimentos e manobras, tanto com o veículo parado como em movimento, e falta de protocolos exclusivos para prevenção e controle de infecção. ${ }^{2}$

Em seu estudo Nascimento e Araújo (2017) expõem que os profissionais que trabalham em APH, por estarem frequentemente expostos a diversos riscos ocupacionais, estão mais propensos ao sofrimento de acidentes e aquisição de patologias e agravos. ${ }^{3}$

Os riscos ocupacionais são decorrentes de fatores específicos do ambiente e condições de trabalho, assim como, características como o tempo de serviço, formação, categoria profissional. Entre os riscos ocupacionais citam-se o contato com fluidos como sangue e secreções, microrganismos como vírus e bactérias, contato com material perfuro - cortante, entre outros geradores de acidentes de trabalho durante o desenvolvimento das atividades laborais. ${ }^{4}$

Segundo o Ministério da Saúde do Brasil, os riscos mais frequentes nos ambientes de trabalho, por categoria, são: físicos (ruídos, temperaturas extremas, iluminação, radiação ionizante e não ionizante), químicos (substâncias químicas que podem estar presentes nos ambientes de trabalho na forma de poeiras, fumos, névoas, neblinas, gases ou vapores), mecânicos (máquinas com partes móveis 


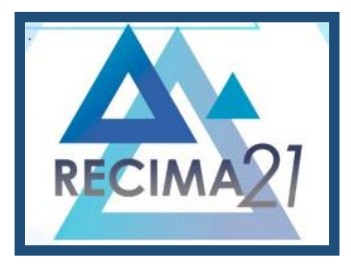

\section{RECIMA21 - REVISTA CIENTÍFICA MULTIDISCIPLINAR ISSN 2675-6218}

não protegidas; calandras e cilindros; guilhotinas; prensas e o uso de instrumentos cortantes ou perfurantes etc.), biológicos (micro-organismos, como bactérias, fungos, protozoários, vírus, entre outros; e animais peçonhentos, como cobras, escorpiões, aranhas), e psicossociais (jornadas de trabalho longas, esforços físicos exagerados com posturas forçadas e carregamento de peso, ritmo acelerado, trabalho repetitivo e monótono; trabalho em turnos e noturno; desemprego; vínculos precários ou ausência de vínculo trabalhista). ${ }^{5}$

Desse modo, o uso de EPI's durante a realização dos procedimentos são recomendações que devem ser seguidas, visto que a adoção dessa medida visa à proteção do profissional, da equipe e dos pacientes, reduzindo a ocorrência de acidentes laborais e infecções nosocomiais, além de evitar consequências às instituições, como os prejuízos institucionais. ${ }^{6}$

Porém, de acordo com Neves (2011) alguns profissionais de saúde justificam não fazer uso dos equipamentos de proteção pela interferência na habilidade e execução de alguns procedimentos realizados em ritmo acelerado, escolhendo assim por não utilizá-los, o que os torna muito mais vulneráveis a exposição de riscos ocupacionais e acidentes de trabalho. ${ }^{7}$

Para Pereira, (2019) outro fator que contribui significativamente para a ocorrência de acidentes de trabalho na enfermagem, é devido à precariedade das condições de trabalho e segurança, resultando em agressões a saúde ou perturbações funcionais, que podem culminar em incapacidades ou até o óbito do indivíduo. ${ }^{8}$ Podendo estar atrelado a falta de preparo e capacitação; sobrecarga de papéis; longas jornadas de trabalho e recursos e insumos insuficientes, sendo estes considerados os riscos rotineiros presentes na área de enfermagem. ${ }^{9}$

Diante do exposto, surge como uma questão norteadora do estudo: Quais as evidências científicas acerca dos riscos ocupacionais da equipe de enfermagem no serviço móvel de urgência? Assim pode-se observar como objetivo analisar as evidências científicas acerca dos riscos ocupacionais dos profissionais da equipe de enfermagem no SAMU.

A realização do estudo relacionado com a temática é de suma importância tanto para acadêmicos de enfermagem quanto para os profissionais de enfermagem do SAMU, haja vista em descrever os principais tipos de riscos ocupacionais que a equipe de enfermagem do SAMU enfrenta diariamente. A pesquisa trará contribuições tanto no sentido de ampliar e fortalecer as discussões acerca da temática proposta, como minimizar tais riscos.

\section{METODOLOGIA}

O presente estudo trata-se de uma pesquisa bibliográfica de natureza qualitativa, utilizando como método a revisão integrativa. Tal método, como define Torraco (2016), é uma forma distinta de pesquisa que possibilita a geração de novos conhecimentos sobre um determinado tema, bem como a revisão de tópicos emergentes. Por meio dela, é possível analisar criticamente e sintetizar a literatura sobre um tópico específico de forma integrada, a fim de que novas perspectivas sejam geradas. ${ }^{10}$ 


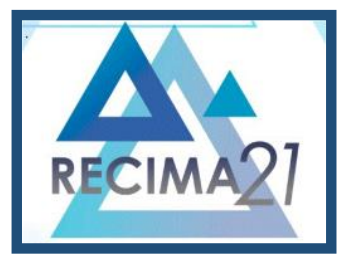

\section{RECIMA21 - REVISTA CIENTÍFICA MULTIDISCIPLINAR ISSN 2675-6218}

Adotou-se a revisão integrativa da literatura por seu perfil sistematizado na busca de produções e na análise dos resultados, visando à compreensão do tema proposto, a partir dos estudos realizados.

${ }^{11} \mathrm{Na}$ qual a pergunta norteadora do processo revisional foi construída por meio da estratégia PICo ( $\mathrm{P}=$ população; $\mathrm{I}=$ interesse ; $\mathrm{Co}=$ contexto) e constitui em: Quais as evidências científicas acerca dos riscos ocupacionais da equipe de enfermagem no SAMU? A estratégia PICo normalmente é usada para elaboração de estudos não clínicos. Esses acrônimos podem ser usados para desenvolvimento de questões de variadas, possibilitando a definição correta de que informações são necessárias para a resolução da questão de pesquisa, além de maximizar a recuperação de evidências das diversas bases de dados, foca o escopo da pesquisa e evita a realização de buscas desnecessárias. ${ }^{12}$

Figura 1: Descrição da Estratégia PICo, Teresina, 2021.

\begin{tabular}{|c|l|l|}
\hline $\mathbf{P}$ & População & Equipe de enfermagem \\
\hline $\mathbf{I}$ & Interesse & Riscos Ocupacionais \\
\hline Co & Contexto & Equipe de enfermagem do SAMU \\
\hline
\end{tabular}

Fonte: Microsoft Offce Excel (2021).

O início do levantamento da pesquisa foi realizado no mês de agosto de 2020, por meio de consultas em publicações científicas nas bases de dados: LILACS (Literatura Latino Americana e do Caribe em Ciências de Saúde); Scielo (Scientific Electronic Library Online); MEDLINE (Medical Literature Analysis and Retrieval System Online); Google scholar e BVS (Biblioteca Virtual em Saúde). Foram utilizados os descritores aplicados, seguidos os Descritores em Ciência e Saúde (DeCS) e o Medical Subject Heading (MeSH): "Riscos ocupacionais" (Occupational risks), "Serviços

Médicos de Emergência" (Emergency medical services); "Equipe de Enfermagem" (Nursing team); onde foi conectado ao operador booleano "OR", após os achados das amostras dos artigos aderiu-se ao cruzamento com o operador booleano "AND". Os critérios de inclusão foram os artigos que abordassem os riscos ocupacionais; publicações com textos completos; publicados entre 2014 e 2019 e disponíveis nos idiomas Português, Inglês e Espanhol. E, os critérios de exclusão: produções incompletas, indisponíveis gratuitamente; teses; dissertações; artigos que não abordassem a temática desse estudo. E após a busca, obteve-se uma amostra de 13 artigos coletados. Observado no fluxograma a seguir: 


\section{RECIMA21 - REVISTA CIENTÍFICA MULTIDISCIPLINAR ISSN 2675-6218}

RISCOS OCUPACIONAIS DA EQUIPE DE ENFERMAGEM NO SERVIÇO DE ATENDIMENTO MÓVEL DE URGÊNCIA Jeane Santos Monteiro, Silvia Vitoria Leal Moreira Reis, Magda Rogéria Pereira Viana

Figura 2. Fluxograma contendo os operadores booleanos utilizados.

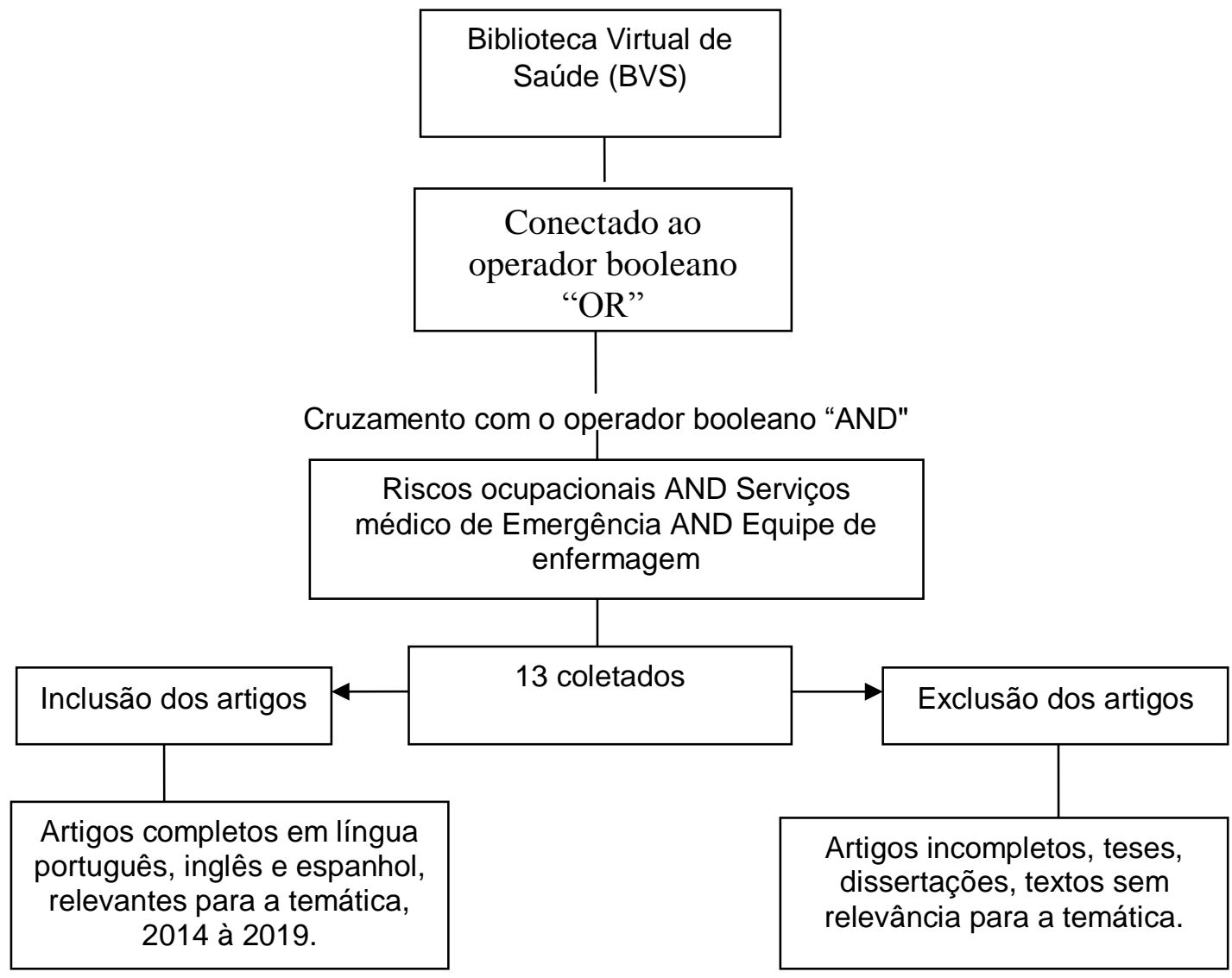

Fonte: pesquisa direta, 2021.

Seguiram-se as seguintes etapas para realizar a análise dos dados: primeiro a pergunta da pesquisa; segundo a pesquisa bibliográfica; terceiro a definição dos critérios de inclusão e exclusão; quarta a análise crítica dos achados. 


\section{RECIMA21 - REVISTA CIENTÍFICA MULTIDISCIPLINAR ISSN 2675-6218}

RISCOS OCUPACIONAIS DA EQUIPE DE ENFERMAGEM NO SERVIÇO DE ATENDIMENTO MÓVEL DE URGÊNCIA Jeane Santos Monteiro, Silvia Vitoria Leal Moreira Reis, Magda Rogéria Pereira Viana

Figura 3- Fluxograma de seleção dos estudos, elaborado a partir do modelo PRISMA.

Teresina, Brasil, 2021.
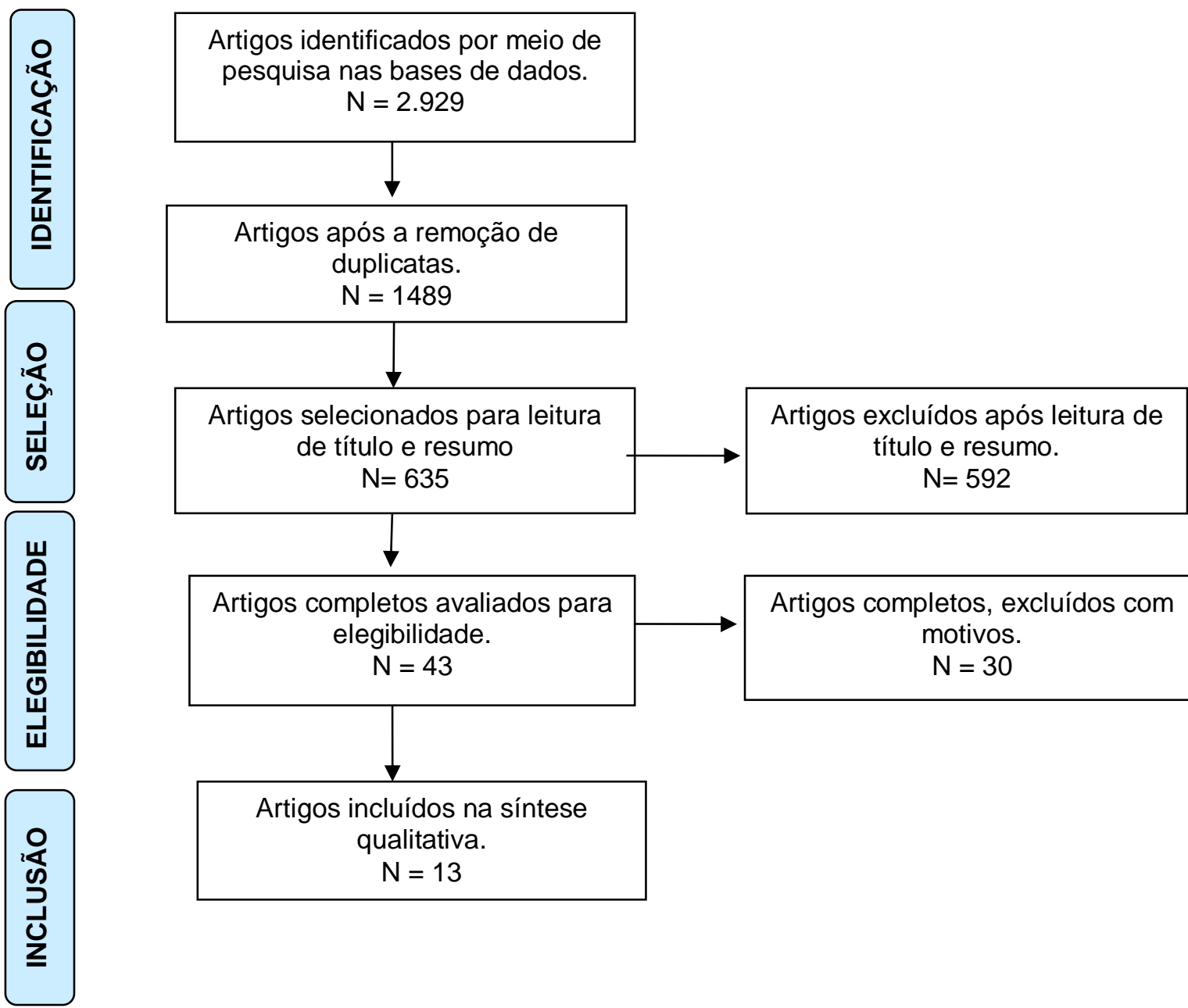

Artigos completos, excluídos com motivos.

$\mathrm{N}=30$

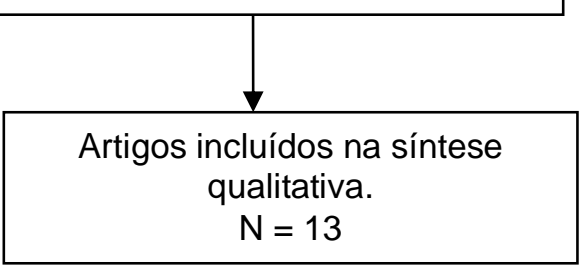

Fonte: PRISMA = PreferredReportingltems for SystematicReviewsand Meta-Analyses .

\section{RESULTADOS}

Diante da estratégia de busca, os autores delinearam variáveis para descrever as evidências encontradas na pesquisa. Descreve-se que a amostra final foi composta por 13 artigos que atendiam aos critérios de inclusão previamente estabelecidos. Na qual, quatro foram extraídos da BVS, dois da LILACS, dois da Scielo e dois da PubMed e três do Google Scholar, sendo que oito estavam escritos na língua portuguesa, e três na língua inglesa e dois em espanhol. Quanto ao ano de publicação a maioria dos artigos encontrados foram publicados em anos distintos, com exceção de quatro deles datados em 2014, os demais foram nos respectivos anos de 2015, 2017, 2018 e 2019. 


\section{RECIMA21 - REVISTA CIENTÍFICA MULTIDISCIPLINAR ISSN 2675-6218}

RISCOS OCUPACIONAIS DA EQUIPE DE ENFERMAGEM NO SERVIÇO DE ATENDIMENTO MÓVEL DE URGÊNCIA Jeane Santos Monteiro, Silvia Vitoria Leal Moreira Reis, Magda Rogéria Pereira Viana

Quadro: Amostra final de artigos incluídos na revisão integrativa da literatura sobre riscos ocupacionais de profissionais da equipe de enfermagem do SAMU.

\begin{tabular}{|c|c|c|c|c|}
\hline $\begin{array}{l}\text { Autor (a)/ } \\
\text { Ano/ } \\
\text { Local }\end{array}$ & Título & $\begin{array}{l}\text { Abordagem } \\
\text { Metodológica }\end{array}$ & Objetivo do Estudo & Contribuições \\
\hline $\begin{array}{l}\text { ARAÚJO; } \\
\text { OLIVEIRA } \\
2019 \\
\text { Distrito } \\
\text { Federal }\end{array}$ & $\begin{array}{l}\text { Mapeamento } \\
\text { dos Riscos } \\
\text { Psicossociais } \\
\text { no SAMU/DF }\end{array}$ & $\begin{array}{l}\text { Quanti- } \\
\text { qualitativo. }\end{array}$ & $\begin{array}{lr}\text { Com o objetivo de } \\
\text { mapear os riscos } \\
\text { psicossociais } \\
\text { Serviço no } \\
\text { Atendimento } \\
\text { de Urivel } \\
\text { do Distrito Federal. }\end{array}$ & $\begin{array}{l}\text { Os resultados puderam } \\
\text { embasar discussões para } \\
\text { ações e políticas de } \\
\text { prevenção em saúde e } \\
\text { trabalho. }\end{array}$ \\
\hline $\begin{array}{l}\text { COSTA et al; } \\
2014 \\
\text { Natal-RN }\end{array}$ & $\begin{array}{l}\text { Riscos } \\
\text { ocupacionais } \\
\text { em um serviço } \\
\text { de } \\
\text { atendimento } \\
\text { móvel de } \\
\text { urgência }\end{array}$ & Quantitativo & 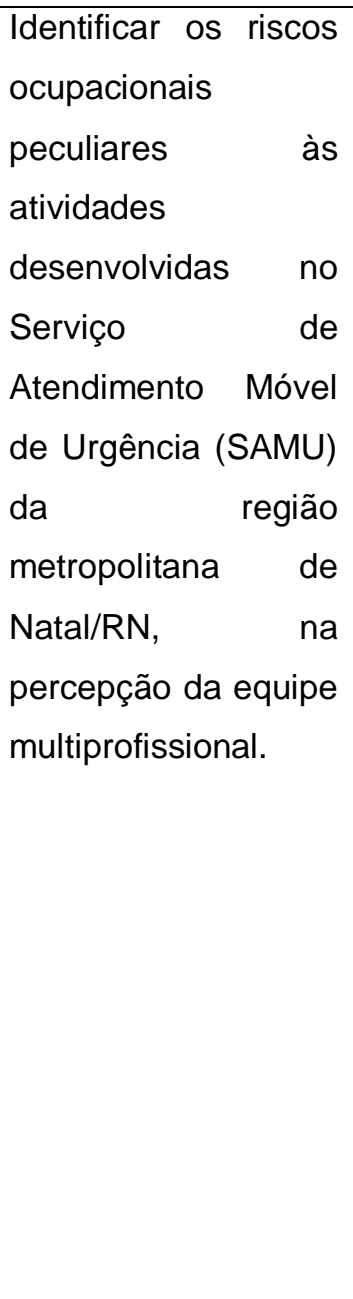 & 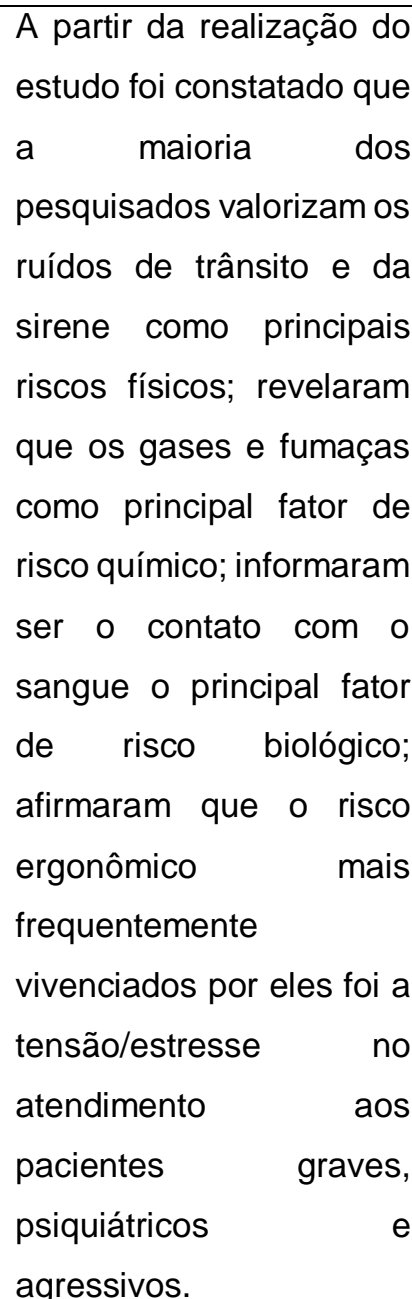 \\
\hline
\end{tabular}




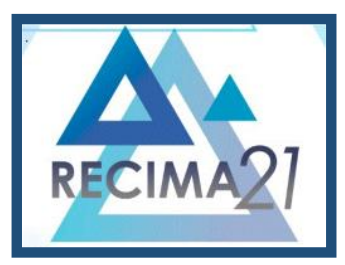

\section{RECIMA21 - REVISTA CIENTÍFICA MULTIDISCIPLINAR ISSN 2675-6218}

RISCOS OCUPACIONAIS DA EQUIPE DE ENFERMAGEM NO SERVIÇO DE ATENDIMENTO MÓVEL DE URGÊNCIA Jeane Santos Monteiro, Silvia Vitoria Leal Moreira Reis, Magda Rogéria Pereira Viana

\begin{tabular}{|c|c|c|c|c|}
\hline $\begin{array}{l}\text { CORREA; } \\
\text { MURILLO \& } \\
\text { CIFUENTES. } \\
2018 \\
\text { Colômbia }\end{array}$ & $\begin{array}{l}\text { Acidentes } \\
\text { com material } \\
\text { biológico em } \\
\text { trabalhadores } \\
\text { da saúde. } \\
\text { Palmira-Valle } \\
\text { del Cauca. } \\
2014-2016\end{array}$ & $\begin{array}{l}\text { Quanti- } \\
\text { qualitativo }\end{array}$ & $\begin{array}{l}\text { Descrever o perfil } \\
\text { dos acidentes de } \\
\text { trabalho por risco } \\
\text { biológico em uma } \\
\text { instituição de saúde } \\
\text { de segundo nível de } \\
\text { complexidade. }\end{array}$ & $\begin{array}{l}\text { Os resultados deste } \\
\text { estudo mostraram que o } \\
\text { tipo de elemento } \\
\text { vulnerável, local da lesão } \\
\text { e a não utilização de EPI } \\
\text { contribui para a definição } \\
\text { do perfil epidemiológico } \\
\text { das lesões. }\end{array}$ \\
\hline $\begin{array}{l}\text { DROPKIN et al } \\
; \\
2015 \\
\text { Great Neck, } \\
\text { NY,EUA }\end{array}$ & $\begin{array}{l}\text { Um estudo } \\
\text { qualitativo de } \\
\text { problemas de } \\
\text { saúde, fatores } \\
\text { de riscos e } \\
\text { prevenção } \\
\text { entre } \\
\text { trabalhadores } \\
\text { de saúde. }\end{array}$ & Qualitativa & $\begin{array}{lr}\text { Identificar } & \text { os } \\
\text { principais problemas } \\
\text { de saúde } \\
\text { relacionados } \\
\text { trabalho ao } \\
\text { trabalhadores da } \\
\text { EMS nos Estados } \\
\text { Unidos; identificar } \\
\text { fatores de risco no } \\
\text { nível organizacional, } \\
\text { tarefa e exposição. }\end{array}$ & $\begin{array}{l}\text { A abordagem da teoria } \\
\text { fundamentada identificou } \\
\text { os principais problemas } \\
\text { de saúde dos } \\
\text { trabalhadores e os } \\
\text { fatores organizacionais e } \\
\text { exposições relacionadas } \\
\text { a eles. }\end{array}$ \\
\hline $\begin{array}{l}\text { MARQUES et } \\
\text { al., } \\
2015 \\
\text { Goiânia - GO }\end{array}$ & $\begin{array}{l}\text { O } \\
\text { absenteísmo } \\
\text { - doença da } \\
\text { equipe de } \\
\text { enfermagem } \\
\text { de um hospital } \\
\text { universitário/ } \\
\text { El absentismo } \\
\text { - } \\
\text { enfermedad } \\
\text { de equipo de } \\
\text { enfermería de } \\
\text { um hospital } \\
\text { universitário. }\end{array}$ & Quantitativa & $\begin{array}{ll}\text { Analisar } & 0 \\
\text { absenteísmo } & - \\
\text { doença apresentado } \\
\text { por profissionais da } \\
\text { equipe de } \\
\text { enfermagem em um } \\
\text { hospital universitário. }\end{array}$ & $\begin{array}{l}\text { Em conclusão do artigo, é } \\
\text { importante adotar } \\
\text { políticas e ações de } \\
\text { saúde que tenham o } \\
\text { trabalhador como foco, } \\
\text { com vistas a assegurar a } \\
\text { promoção da saúde e a } \\
\text { prevenção de agravos } \\
\text { ocupacionais, de modo a } \\
\text { garantir a qualidade da } \\
\text { assistência prestada ao } \\
\text { paciente. }\end{array}$ \\
\hline
\end{tabular}




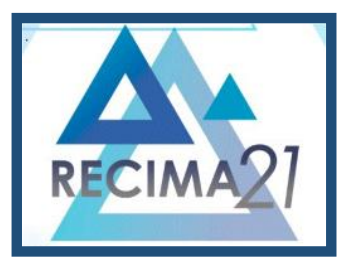

\section{RECIMA21 - REVISTA CIENTÍFICA MULTIDISCIPLINAR ISSN 2675-6218}

\begin{tabular}{|c|c|c|c|c|}
\hline $\begin{array}{l}\text { NASCIMENT } \\
\text { O } \\
2019 \\
\text { Bahia }\end{array}$ & $\begin{array}{l}\text { Riscos } \\
\text { Ocupacionais } \\
\text { dos } \\
\text { Profissionais } \\
\text { de } \\
\text { Enfermagem } \\
\text { atuantes no } \\
\text { SAMU } 192\end{array}$ & Qualitativa & $\begin{array}{l}\text { Avaliar a percepção } \\
\text { de riscos } \\
\text { ocupacionais que os } \\
\text { profissionais } \quad \text { de } \\
\text { enfermagem } \\
\text { atuantes no } \\
\text { atendimento móvel } \\
\text { de urgência. }\end{array}$ & $\begin{array}{l}\text { A identificação dos } \\
\text { fatores de riscos os quais } \\
\text { estão expostos contribui } \\
\text { de forma efetiva para } \\
\text { elaboração de medidas } \\
\text { de proteção necessárias } \\
\text { a fim de minimizar os } \\
\text { riscos ocupacionais }\end{array}$ \\
\hline $\begin{array}{l}\text { PERES; } 2018 \\
\text { Rio Grande do } \\
\text { Sul. }\end{array}$ & $\begin{array}{l}\text { Atuação do } \\
\text { enfermeiro em } \\
\text { um serviço de } \\
\text { atendimento } \\
\text { pré-hospitalar } \\
\text { privado. }\end{array}$ & Qualitativa & $\begin{array}{lr}\text { Conhecer } & \text { a } \\
\text { percepção } & \text { de } \\
\text { trabalhadores } & \text { de } \\
\text { saúde sobre } & \text { a } \\
\text { atuação } & \text { do } \\
\text { enfermeiro } & \text { no } \\
\text { serviço } & \text { de } \\
\text { atendimento pré- } \\
\text { hospitalar privado. }\end{array}$ & $\begin{array}{l}\text { Em conclusão foi possível } \\
\text { conhecer a percepção de } \\
\text { trabalhadores de saúde } \\
\text { sobre a atuação do } \\
\text { enfermeiro em um serviço } \\
\text { de atendimento pré } \\
\text { hospitalar. A partir das } \\
\text { vivências teceram-se } \\
\text { algumas considerações } \\
\text { importantes a respeito do } \\
\text { cotidiano de trabalho de } \\
\text { APH, com objetivo de } \\
\text { analisar as } \\
\text { particularidades de } \\
\text { atividade do enfermeiro } \\
\text { nesta área. }\end{array}$ \\
\hline $\begin{array}{l}\text { REICHARD et } \\
\text { al ; } 2017 \\
\text { Estados } \\
\text { Unidos }\end{array}$ & $\begin{array}{l}\text { Lesões } \\
\text { ocupacionais } \\
\text { e exposições } \\
\text { entre } \\
\text { trabalhadores } \\
\text { de serviços } \\
\text { médicos de } \\
\text { emergência. }\end{array}$ & Qualitativa & $\begin{array}{l}\text { Descrever as } \\
\text { circunstâncias das } \\
\text { lesões e exposições } \\
\text { ocupacionais entre } \\
\text { os trabalhadores da } \\
\text { EMS para orientar os } \\
\text { esforços de } \\
\text { prevenção de lesões. }\end{array}$ & $\begin{array}{l}\text { Novos e aprimorados } \\
\text { esforços para prevenir } \\
\text { lesões aos trabalhadores } \\
\text { do SEM são necessários } \\
\text { especialmente aqueles } \\
\text { que visam prevenir } \\
\text { lesões por movimento do } \\
\text { corpo e exposição a } \\
\text { substâncias nocivas. }\end{array}$ \\
\hline $\begin{array}{l}\text { SALVI et al., } \\
2017 \\
\text { Houston }\end{array}$ & $\begin{array}{l}\text { Impactos } \\
\text { psicológicos } \\
\text { da exposição } \\
\text { do } \\
\text { escapamento }\end{array}$ & Qualitativa & \begin{tabular}{l}
\multicolumn{2}{l}{ Determinar o impacto } \\
psicológico do \\
escapamento do \\
veículo em ratos.
\end{tabular} & $\begin{array}{l}\text { Em conclusão, nosso } \\
\text { estudo propõe um } \\
\text { modelo novo } \\
\text { translacionalmente } \\
\text { relevante para investigar }\end{array}$ \\
\hline
\end{tabular}

RECIMA21 - Ciências Exatas e da Terra, Sociais, da Saúde, Humanas e Engenharia/Tecnologia 


\section{RECIMA21 - REVISTA CIENTÍFICA MULTIDISCIPLINAR ISSN 2675-6218}

RISCOS OCUPACIONAIS DA EQUIPE DE ENFERMAGEM NO SERVICCO DE ATENDIMENTO MÓVEL DE URGÊNCIA Jeane Santos Monteiro, Silvia Vitoria Leal Moreira Reis, Magda Rogéria Pereira Viana

\begin{tabular}{|c|c|c|c|c|}
\hline & $\begin{array}{l}\text { de veículos: } \\
\text { percepções } \\
\text { de um modelo } \\
\text { animal. }\end{array}$ & & & $\begin{array}{l}\text { o efeito de exposições } \\
\text { prolongadas a pró- } \\
\text { oxidantes na exaustão de } \\
\text { veículos em ratos em um } \\
\text { ambiente de laboratório. }\end{array}$ \\
\hline $\begin{array}{l}\text { SOUSA; } \\
\text { SOUZA } \\
\text { COSTA } \\
2014 \\
\text { Campina } \\
\text { Grande }\end{array}$ & $\begin{array}{l}\text { Riscos } \\
\text { ocupacionais } \\
\text { no } \\
\text { Atendimento } \\
\text { Pré-Hospitalar } \\
\text { Móvel: } \\
\text { produção } \\
\text { científica em } \\
\text { periódicos } \\
\text { online/ } \\
\text { Occupational } \\
\text { risks in the } \\
\text { Mobile Pre- } \\
\text { Hospital Care: } \\
\text { scientific } \\
\text { production in } \\
\text { on-line } \\
\text { journals. }\end{array}$ & Qualitativa & 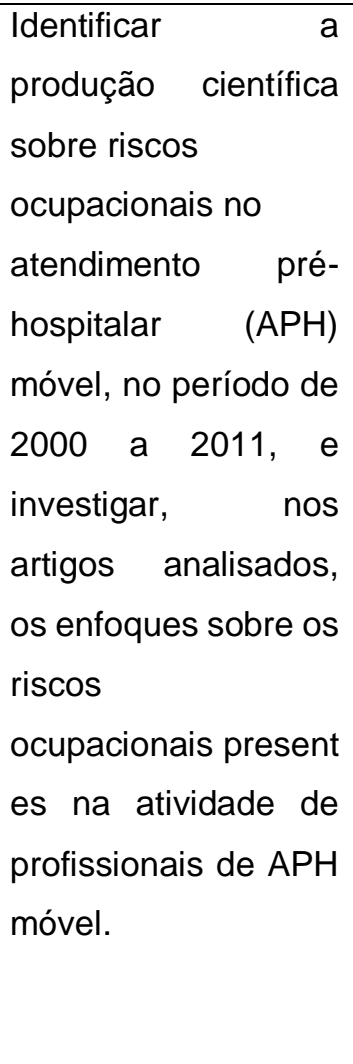 & $\begin{array}{l}\text { Ressalta-se que foi } \\
\text { averiguada } \\
\text { necessidade de políticas } \\
\text { de saúde voltadas para } \\
\text { essa área, em particular, } \\
\text { e condições } \\
\text { trabalho digno, com } \\
\text { lo escopo de amenizar a } \\
\text { insalubridade do APH } \\
\text { móvel. }\end{array}$ \\
\hline $\begin{array}{l}\text { SILVA et al., } \\
2014 \\
\text { Santa Catarina }\end{array}$ & $\begin{array}{l}\text { Riscos de } \\
\text { adoecimento } \\
\text { enfrentados } \\
\text { pela equipe de } \\
\text { enfermagem } \\
\text { do SAMU: } \\
\text { Uma revisão } \\
\text { integrativa }\end{array}$ & Qualitativa & $\begin{array}{l}\text { Foi investigar através } \\
\text { da literatura os riscos } \\
\text { de adoecimento } \\
\text { enfrentados pela } \\
\text { equipe de } \\
\text { Enfermagem do } \\
\text { SAMU }\end{array}$ & $\begin{array}{l}\text { O resultado deste estudo } \\
\text { mostrou que a Equipe de } \\
\text { enfermagem está } \\
\text { inserida em um local que } \\
\text { favorece ao adoecimento, } \\
\text { mas que com o uso dos } \\
\text { Equipamentos de } \\
\text { Proteção Individual esses } \\
\text { riscos poderão ser } \\
\text { afastados. }\end{array}$ \\
\hline
\end{tabular}




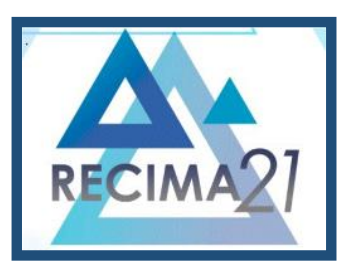

\section{RECIMA21 - REVISTA CIENTÍFICA MULTIDISCIPLINAR ISSN 2675-6218}

RISCOS OCUPACIONAIS DA EQUIPE DE ENFERMAGEM NO SERVIÇO DE ATENDIMENTO MÓVEL DE URGÊNCIA Jeane Santos Monteiro, Silvia Vitoria Leal Moreira Reis, Magda Rogéria Pereira Viana

\begin{tabular}{|c|c|c|c|c|}
\hline $\begin{array}{l}\text { SILVA et al., } \\
2014 \\
\text { Colômbia }\end{array}$ & $\begin{array}{l}\text { O ruído } \\
\text { causando } \\
\text { danos } \\
\text { estresse: } \\
\text { possibilidade } \\
\text { de atuação } \\
\text { para a } \\
\text { enfermagem } \\
\text { do trabalho. }\end{array}$ & Qualitativa & $\begin{array}{l}\text { Identificar os riscos } \\
\text { ocupacionais que } \\
\text { acometem } \\
\text { trabalhadores que } \\
\text { atuam em } \\
\text { ambientes ruidosos, } \\
\text { discutindo } \\
\text { consequências à à } \\
\text { saúde } \\
\text { trabalhador. }\end{array}$ & $\begin{array}{l}\text { É necessário uma } \\
\text { transformação } \\
\text { progressiva na } \\
\text { organização e nas } \\
\text { condições e processo de } \\
\text { trabalho, através de plano } \\
\text { de ações de avaliação } \\
\text { somado ao processo } \\
\text { educativo contínuo. Além } \\
\text { disso, o planejamento da } \\
\text { assistência de } \\
\text { enfermagem deve } \\
\text { envolver a prevenção, } \\
\text { promoção da saúde e } \\
\text { qualidade de vida no } \\
\text { ambiente de trabalho. }\end{array}$ \\
\hline $\begin{array}{l}\text { WEAVER et } \\
\text { al., } \\
2015 \\
\text { Boston }\end{array}$ & $\begin{array}{l}\text { Um estudo } \\
\text { observacional } \\
\text { da duração do } \\
\text { turno, } \\
\text { familiaridade } \\
\text { da tripulação e } \\
\text { lesões } \\
\text { doenças } \\
\text { ocupacionais } \\
\text { em } \\
\text { trabalhadores } \\
\text { de serviços } \\
\text { médicos de } \\
\text { emergência; }\end{array}$ & Qualitativa & $\begin{array}{l}\text { Avaliar o impacto da } \\
\text { duração do turno nos } \\
\text { relatórios internos de } \\
\text { lesões e doenças } \\
\text { ocupacionais em um } \\
\text { corte nacional de } \\
\text { trabalhadores de } \\
\text { EMS. }\end{array}$ & $\begin{array}{l}\text { Em conclusão do estudo, } \\
\text { conclui-se que a duração } \\
\text { prolongada do turno esta } \\
\text { associada a lesões e } \\
\text { doenças ocupacionais } \\
\text { entre os trabalhadores } \\
\text { por turno do EMS. }\end{array}$ \\
\hline
\end{tabular}

Fonte: pesquisa direta (2021).

\section{DISCUSSÃO}

\section{Risco Físico}

Segundo a Norma Regulamentadora NR5, são considerados riscos físicos: ruídos, vibrações, radiações, temperaturas extremas, pressão anormal e umidade. Dentre os tipos de risco físicos 


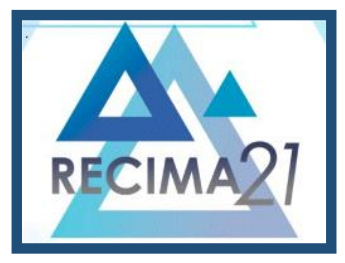

\section{RECIMA21 - REVISTA CIENTÍFICA MULTIDISCIPLINAR ISSN 2675-6218}

RISCOS OCUPACIONAIS DA EQUIPE DE ENFERMAGEM NO SERVIÇO DE ATENDIMENTO MÓVEL DE URGÊNCIA Jeane Santos Monteiro, Silvia Vitoria Leal Moreira Reis, Magda Rogéria Pereira Viana

presentes no APH encontram-se aqueles relacionados com intempéries (calor, frio e chuva), umidades, ruídos e vibrações. ${ }^{13}$

A literatura aponta dentro da categoria de riscos físicos os ruídos como o mais frequente na equipe de enfermagem por conta da necessidade do uso da sirene da ambulância. ${ }^{14}$

Tal risco pode causar a surdez, um dos distúrbios mais evidentes nos indivíduos expostos ao som de sirene de ambulância. No entanto, existem outros riscos provocados pelo ruído como: estresse no atendimento ao paciente, dificuldade de concentração, dispersão, fatores que podem comprometer a saúde do trabalhador, levando ao absenteísmo. ${ }^{15}$

\section{Risco Químico}

Segundo Silva et al., (2014) os produtos químicos no atendimento pré-hospitalar estão ligados a procedimentos de esterilização, desinfecção e ao tratamento medicamentoso dos pacientes. Podem entrar em contato com o organismo por exposição crônica ou acidental, podendo ocasionar explosões e incêndios, além de contaminação, que pode levar ao aparecimento de efeitos carcinogênicos, teratogênicos, asfixiantes, entre outros. ${ }^{16}$

O autor supracitado relata que os profissionais entram em contato com substâncias químicas através da manipulação de medicamentos, uso de desinfetantes e outros produtos químicos, ou de forma acidental. Partes das consequências advindas desses incidentes podem ser amenizadas pelo uso apropriado dos EPI's.

No entanto, outra pesquisa relata que os gases e a fumaça são os principais riscos encontrados nessa categoria. Os gases podem desencadear irritações nos tecidos expostos a longo tempo, podendo ainda ter ação depressiva no sistema nervoso central, além do mais, podendo agir também como asfixiante ocasionando redução na concentração de oxigênio tecidual. ${ }^{17}$

E ainda, em experiências realizadas nos Estados Unidos da América com ratos em laboratórios, com o objetivo de investigar os efeitos da exposição prolongada às emissões dos escapes de veículos, os pesquisadores observaram alterações das funções comportamentais e cognitivas, tais como ansiedade, depressão e memória prejudicada. ${ }^{18}$

\section{Risco Mecânico}

De acordo com Sousa, Souza e Costa (2014), os riscos mecânicos devem-se, principalmente, ao fato da necessidade de uma ação rápida do profissional, o que pode levar à desatenção ao descartar a agulha e em dificuldade em puncionar pacientes agitados. ${ }^{19}$

\section{Risco Biológico}




\section{RECIMA21 - REVISTA CIENTÍFICA MULTIDISCIPLINAR ISSN 2675-6218}

RISCOS OCUPACIONAIS DA EQUIPE DE ENFERMAGEM NO SERVIÇO DE ATENDIMENTO MÓVEL DE URGÊNCIA Jeane Santos Monteiro, Silvia Vitoria Leal Moreira Reis, Magda Rogéria Pereira Viana

Um estudo com 161 funcionários que trabalham em ambulâncias verificou que quando trata-se do sangue, há uma maior exposição a pacientes com suspeita ou confirmação de Hepatites Virais e com o Vírus da Imunodeficiência Humana (HIV) através de contato direto com o sangue dos mesmos, por acidente com agulhas ou outros materiais perfurocortantes. Os dados das entrevistas revelaram que $95 \%$ dos profissionais usavam luvas descartáveis regularmente ao entrar em contato com sangue, fluidos corporais ou materiais potencialmente infecciosos. Aproximadamente $84,5 \%$ lavavam regularmente suas mãos antes e depois de ter contato com sangue, fluidos corporais ou materiais contaminados. Cerca de $82,6 \%$ usavam luvas descartáveis ao fazer punções de sangue ou administração de fluido. Apenas $54 \%$ romperam os frascos de drogas usando um pano limpo ou algodão como recomendado para proteger de lesão. A pontuação média para as práticas preventivas foi 7,6 $\pm 2,2$ (pontuação total =10), uma nota moderada, porém, observou-se que apenas 30\% utilizavam equipamentos de proteção de maneira adequada. ${ }^{20}$

Correa, Murillo e Cifuentes (2018) identificaram em seu estudo que $89 \%$ dos trabalhadores expostos ao risco biológico não usavam materiais ou equipamentos de segurança no momento do acidente. A manipulação de agulhas e os movimentos dos pacientes ao realizar procedimentos de atendimento foram as causas mais frequentes. ${ }^{21}$

Nesse sentido Sousa, Souza e Costa (2014) afirmam que muitos acidentes com riscos biológicos envolvendo profissionais do SAMU estão diretamente relacionados com a não adesão ou uso inadequado dos EPl's, e que o contato com fluídos corpóreos é um risco frequente nas atividades desses profissionais, e esse risco se torna altamente potencial quando o profissional não utiliza os EPI's adequados. ${ }^{19}$

\section{Risco Ergonômico/ psicossocial}

Segundo Dropkin et al.,( 2015) a ergonomia da unidade de trabalho (ambulância) gera condições de exposição a riscos de lesões músculo esqueléticas. A permanência sentada no interior do veículo entre as chamadas de atendimentos e a necessidade de respostas musculares rápidas quando acionadas, são relatadas como causadoras de lesões e adoecimento com frequência. ${ }^{22}$

Estudos com 572 trabalhadores do Emergency Medical Services (SEM) identificaram entre as lesões sofridas pelos trabalhadores: as entorses e distensões, lesões corporais decorrentes do levantamento, transporte e transferência de pacientes e equipamentos e as exposições a substâncias nocivas à saúde. ${ }^{23}$

Entre os riscos ergonômicos, destaca-se a jornada de trabalho prolongada, que é característica do processo de trabalho desenvolvido, representando um aumento na exposição aos acidentes de trabalho e adoecimento dos profissionais que desenvolvem suas funções laborais em jornadas de mais ou menos 8 horas de trabalho, apresentam menos risco de agravos à saúde quando comparado aos que possuem jornadas maiores que 8 horas e menores que 12 horas. ${ }^{24}$ 


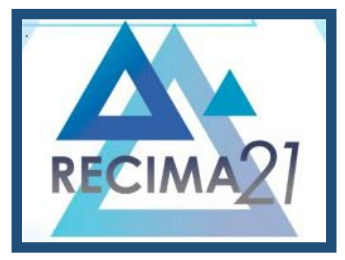

\section{RECIMA21 - REVISTA CIENTÍFICA MULTIDISCIPLINAR ISSN 2675-6218}

Para prevenir o desenvolvimento de lesões é essencial que os profissionais tenham conhecimentos sobre os riscos ergonômicos a que estão suscetíveis. Com isso, os enfermeiros do trabalho devem promover atividades com teor educativo e preventivo, incentivando os profissionais a novos hábitos, reeducação postural, bem como a realização de pausas durante o dia, respeitando seu tempo de descanso (MARQUES et al., 2015). ${ }^{25}$

De acordo com Araújo; Oliveira, (2019) os riscos psicossociais podem ser entendidos como frutos dos efeitos negativos do estilo de gestão e organização de trabalho, dos danos físicos sofridos, psicológicos e também sociais, os quais levam os profissionais ao adoecimento com consequente comprometimento na qualidade do trabalho. ${ }^{26}$

\section{CONSIDERAÇÕES FINAIS}

De acordo com a análise mais aprofundada dos artigos, constatou-se que a equipe de enfermagem do SAMU sofre diariamente com os riscos ocupacionais presentes em seu ambiente de trabalho. Dentre os riscos analisados, o mais frequente na literatura foi o risco ergonômico/ psicossocial, em seguida o risco biológico. Isso mostra que existe a necessidade de elaborar políticas públicas voltadas para a prevenção dos mesmos nos serviços médicos de emergência a fim de reduzir acidentes com esses profissionais.

Verificou-se que as publicações acerca dos riscos ocupacionais no âmbito pré-hospitalar móvel aconteceram em maior número no ano de 2014. O principal delineamento metodológico utilizado foi o qualitativo e os enfermeiros foram os profissionais que estiveram presentes na maioria das publicações.

Como limitação do estudo, observa-se a dificuldade de encontrar pesquisas com abordagem do risco mecânico com o intuito de fomentar maior conhecimento de como esse risco interfere na atividade laboral e na saúde dos profissionais da equipe de enfermagem.

Adjunto a isto, torna-se essencial à elaboração de novos estudos que abordem essa temática a fim de minimizar tais riscos inerentes a essa área de atuação, com enfoque principalmente nos riscos de maior prevalência.

\section{REFERÊNCIAS}

Brasil. Ministério da Saúde. Comissão de intergestores Tripartite.Portaria n.ำ 2048/GM, 05 de de Novembro de 2002[internet]. Brasília: ministério da saúde; 2002 [ cited 2020 agosto 15]. Available from: https://bvsms.saude.gov.br/bvs/saudelegis/gm/2002/prt2048 0511 2002.html

Araújo L.R, Moreira M.R. Risco ocupacional enfrentado pela equipe de enfermagem do serviço de atendimento móvel de urgência. 2015. 3 p. 2 vol.

Nascimento M.O Nascimento, Araújo G.F. Riscos ocupacionais dos profissionais de enfermagem atuantes no SAMU 192. ID on line REV de Psicologia [Internet]. 2017 [cited 2021 May 1];10(33):212223. DOI: https://doi.org/10.14295/idonline.v10i33.614. Available from: https://idonline.emnuvens.com.br/id/article/view/614 


\section{RECIMA21 - REVISTA CIENTÍFICA MULTIDISCIPLINAR ISSN 2675-6218}

RISCOS OCUPACIONAIS DA EQUIPE DE ENFERMAGEM NO SERVIÇO DE ATENDIMENTO MÓVEL DE URGÊNCIA Jeane Santos Monteiro, Silvia Vitoria Leal Moreira Reis, Magda Rogéria Pereira Viana

COUTO PLS et al. Representações sociais acerca dos riscos de acidentes de trabalho. Rev Bras Promoç Saúde [Internet]. 2018 [citado 2021 abr. 28];31(2):1-10. DOI: 10.5020/18061230.2018.7074. Available from: https://periodicos.unifor.br/RBPS/article/view/7074.

BRASIL. Ministério da Saúde. Secretaria de Atenção à Saúde. Secretaria de Vigilância em Saúde. Saúde do trabalhador e da trabalhadora [recurso eletrônico]. Cadernos de Atenção Básica, n. 41.

Brasília: Editora do Ministério da Saúde, 2018. [ cited 2020 August 18]. Available from: http://189.28.128.100/dab/docs/portaldab/publicacoes/cadernoab saude do trabalhador.pdf

Porto J.S, Marziale M.H.P. Motivos e consequências da baixa adesão às precauções padrão pela equipe de enfermagem. Rev Gaúcha de Enferm [Internet]. 2016 [cited 2021 Apr 17];37(2):57395. DOI: https://doi.org/10.1590/1983-1447.2016.02.57395. Available from: https://www.scielo.br/scielo.php?pid=S1983-14472016000200501\&script=sci arttext

Neves H.C.C, Souza A.C.S, Medeiros M., Munari D.B, Ribeiro L.C.M, Tipple A.F.V. Segurança dos trabalhadores de enfermagem e fatores determinantes para adesão aos equipamentos de proteção individual. Rev Latino Am Enfermagem [Internet]. 2011 [cited 2021 Apr 29];19(2):354-361. DOI https://doi.org/10.1590/S0104-11692011000200018. Available from: https://www.scielo.br/scielo.php?pid=S0104-11692011000200018\&script=sci abstract\&tlng=pt

Pereira J.A.S, Oliveira A.A.L, Araújo B.P.L, Eloi A.C. The Nurses' Role With Regards to the Risks and Vulnerabilities Faced by Civil Construction Workers / Papel do Enfermeiro Sobre os Riscos e Vulnerabilidades de Trabalhadores da Construção Civil. Rev de Pesquisa: Cuidado é Fundamental Online [Internet]. 2019 [cited 2021 Apr 28];11(3):780-789. DOI: https://doi.org/10.9789/21755361.2019.v11i3.780-784. Available from: http://www.seer.unirio.br/index.php/cuidadofundamental/article/view/6915

Fortini R.G, Saboia V.M, Gomes D.F, Ferreira A.M.O. O esgotamento físico dos enfermeiros no setor de urgência e emergência: revisão integrativa. Rev Nursing [Internet]. 2019 [cited 2021 May 25];v.251:p.2839-2843. Available from: http://www.revistanursing.com.br/revistas/251/pg26.

Torraco R.J. Writing Integrative Literature Reviews: Using the Past and Present to Explore the Future [Internet]. 4th Rev. ed. Human Resource Development Review: [publisher unknown]; 2016 [cited 2021 Apr 29]. 404-428. p. 15 vol. DOI ttps://doi.org/10.1177/1534484316671606. Available from: https://journals.sagepub.com/doi/abs/10.1177/1534484316671606

Ganong L.H. Integrative reviews of nursing research. Rev Nurs Health. 1987;10(1):1-11.

Lockwood C., et al. Systematic reviews of qualitative evidence. In: Aromataris E, Munn Z. Joanna Briggs Institute Reviewer's Manual: The Joanna Briggs Institute. 2017;(Chapte 2).

Peres P.S.Q, Arboit E.L, Camponogara S., Pilau C.O.B, Menezes L.P, Kaefer C.T. Atuação do enfermeiro em um serviço de atendimento pré-hospitalar privado / Nurse performance on a private prehospital assistance. Rev de Pesquisa: Cuidado é Fundamental Online [Internet]. 2018 [cited 2021 Apr 23];10(2) DOI: $\quad$ https://doi.org/10.9789/2175-5361.2018.v10i2.413-422. Available from: http://seer.unirio.br/index.php/cuidadofundamental/article/view/6064

Leite H. D. C. S., Carvalho M. D., Cariman S. D. S., Araújo E. D. M, Silva N. C., Carvalho A. D. O. Risco ocupacional entre profissionais de saúde do Serviço de Atendimento Móvel de Urgência-SAMU. Enferm. Foco [Internet]. 2016 [cited 2021 May 2]; 7(4):31-35. Available from: http://revista.portalcofen.gov.br/index.php/enfermagem/article/viewFile/912/342

Silva J.L, Costa F.S, Souza R.F, Sousa J.L, Oliveira R.S. O ruído causando danos e estresse: possibilidade de atuação para a enfermagem. Avances en Enfermería [Internet]. 2014 [cited 2021 May 2]; 32(1):124-138. DOI: https://doi.org/10.15446/av.enferm.v32n1.46074. Available from: https://revistas.unal.edu.co/index.php/avenferm/article/view/46074 


\section{RECIMA21 - REVISTA CIENTÍFICA MULTIDISCIPLINAR ISSN 2675-6218}

RISCOS OCUPACIONAIS DA EQUIPE DE ENFERMAGEM NO SERVIÇO DE ATENDIMENTO MÓVEL DE URGÊNCIA Jeane Santos Monteiro, Silvia Vitoria Leal Moreira Reis, Magda Rogéria Pereira Viana

Silva O.M, Ascari R.A, Amora R, Schivianato D, Ribeiro M.C. Riscos de adoecimento enfrentados pela equipe de enfermagem do SAMU: uma revisão integrativa / Risks of illness facing the nursing team of SAMU: an integrative review. Rev. Saúde Pública St. Catarina. 2014 jan.-abr;7(1):107-121. Available from: https://pesquisa.bvsalud.org/portal/resource/pt/biblio-1130162

Costa I.K.F, Liberato S.M.D, Costa I.K.F, Melo M.D.M, Simpson C.A, Farias G.M. Riscos ocupacionais em um serviço de atendimento móvel de urgência. Revista de Pesquisa Cuidado é Fundamental Online [Internet]. 2014 [cited 2021 Apr 10];6(3):938-947. DOI: 10.9789/2175-5361.2014v6n3p938. Available from: https://www.redalyc.org/articulo.oa?id=505750623009

Salvi A., Patki G., Liu H., Salim S. Psychological Impact of Vehicle Exhaust Exposure: Insights from an Animal Model. Scientific Reports [Internet]. 2017 [cited 2021 Apr 18];7(1) DOI https://doi.org/10.1038/s41598-017-08859-1. Available from: https://www.nature.com/articles/s41598$\underline{017-08859-1}$

Souza E.R, Sousa A.T.O, Costa I.C.P. Riscos Ocupacionais No Atendimento Pré-Hospitalar Móvel: Produção Científica Em Periódicos Online. RBCS [Internet]. 23ํ de março de 2014 [citado 2 de maio de 2021];18(2):151-6. Available from: https://periodicos.ufpb.br/index.php/rbcs/article/view/15654

Luksamijarulkul P., Pipitsangjan S., Vatanasomboon P. Risk towards blood-borne infections among ambulance personnel in a provincial hospital network in Thailand. Southeast Asian Journal of Tropical Medicine and Public Health [Internet]. 2014 [cited 2021 Apr 28];45(4):940-948. DOI: http://dx.doi.org/10.22463/17949831.1408. https://dialnet.unirioja.es/servlet/articulo?codigo $=7490941$

Available

from:

Correa P.A.A, Medina J.J.M, Cifuentes M.S. Acidentes com material biológico em trabalhadores da saúde. Palmira-Valle del Cauca. 2014-2016. Rev. cienc. cuidad [Internet]. 2018 [cited $2021 \mathrm{Apr}$ 28];15(2):140-150. DOI DOI: http://dx.doi.org/10.22463/17949831.1408. Available from: https://dialnet.unirioja.es/servlet/articulo?codigo $=7490941$

Dropkin J., Moline J., Power P.M, Kim H. A qualitative study of health problems, risk factors, and prevention among Emergency Medical Service workers. Work [Internet]. 2015 [cited 2021 Apr 20];52(4):935-951. DOI: 10.3233/WOR-152139. Available from: https://pubmed.ncbi.nlm.nih.gov/26409382/.

Reichard A.A, Marsh S.M, Tonozzi T.R, Konda S., Gormley M.A. Occupational injuries and exposures among emergency medical services workers. Taylor and Francis Online [Internet]. 2017 [cited 2021 Apr 15];21(4):420-431. DOI: https://doi.org/10.1080/10903127.2016.1274350. Available from: https://www.tandfonline.com/doi/abs/10.1080/10903127.2016.1274350

Weaver M.D, Patterson D.P, Fabio Anthony, Moore C.G, Freiberg M.S, Songer T.J. An observational study of shift length, crew familiarity, and occupational injury and illness in emergency medical services workers. Workplace [Internet]. 2015 [cited 2021 May 3]; doi:10.1136/oemed-2015-102966. Available from: https:// www.ncbi.nlm.nih.gov/pubmed/26371071

Marques D.O, Pereira M.S, Souza A.C.S, Vila V.S.C, Almeida C.C.O.F, Oliveira E.C. O absenteísmo doença da equipe de enfermagem de um hospital universitário. Rev. Bras. Enferm [Internet]. 2015 [cited 2021 May 7];68(5):876-882. DOI: https://doi.org/10.1590/0034-7167.2015680516i. Available from: https://www.scielo.br/j/reben/a/fcTJ5HfwQwmTztXd8KgCbn

Araújo L.K.R, Oliveira S.S. Mapeamento dos Riscos Psicossociais no SAMU/DF. Psicol. cienc. prof [Internet]. 2019 [cited 2021 May 5];39 DOI: https://doi.org/10.1590/1982-3703003184126. Available from: https://www.scielo.br/j/pcp/a/bsWV5KMwctDbgWHCSgd7k5v/?lang=pt 